\title{
Do gold rings protect against articular erosion in rheumatoid arthritis?
}

\author{
Diarmuid M Mulherin, George R Struthers, R Deva Situnayake
}

\begin{abstract}
Objective-To examine the hypothesis that gold rings might delay articular erosion at the metacarpophalangeal (MCP) joint of the left ring finger in ring wearers with rheumatoid arthritis (RA). Methods-Consecutive patients with RA were recruited. They were classified as ring wearers if they had worn a gold ring on the left ring finger throughout most of the time since disease onset, or as non-ring wearers if they had never worn a gold ring. Standard hand radiographs (with rings removed, where possible) were taken and articular erosion was quantified at the MCP and proximal interphalangeal joints.
\end{abstract}

Results-Thirty ring wearers (27 female) and 25 non-ring wearers (12 female) were included. The median ( 25 th-75th centile) Larsen score in the left hand ring MCP joint of ring wearers was 1.0 (1.0-2.0), which was significantly less than in their equivalent right hand joint $(1.0,1.0-5.0, p$ $=0.01$ ). It also tended to be less than the equivalent left hand joint of non-ring wearers $(4.0,1.0-5.0, p=0.06)$, with $a$ similar but significant difference observed at the adjacent middle finger MCP joint ( $p$ $=0.01$ ).

Conclusions-The results of this preliminary study suggest that there may be less articular erosion at the left hand ring, and perhaps adjacent, MCP joints observed in ring wearers with RA. These data support the hypothesis that gold could pass from a gold ring through skin and local lymphatics 'downstream' to nearby MCP joint in sufficient quantities to delay articular erosion.

(Ann Rheum Dis 1997;56:497-499)

Rheumatology, City

Hospital NHS Trust,

Birmingham

D M Mulherin

R D Situnayake

Department of Rheumatology, Coventry and Warwickshire Hospital, Coventry

G R Struthers

Correspondence to:

Dr R D Situnayake,

Department of

Rheumatology, City Hospital

NHS Trust, Dudley Road,

Birmingham $\mathrm{B} 18 \mathrm{QH}$.

Accepted for publication 22 May 1997 symmetrical and sparing of a joint that might otherwise be expected to be involved attracts interest, as we seek to understand the ence the clinical and laboratory manifestations of synovial inflammation, their ability to delay or prevent articular erosion is uncertain. ${ }^{2}$ Articular involvement in RA is usually mechanisms involved in promoting, or preventing, the development of articular erosion. ${ }^{34}$

We observed that the left ring finger metacarpophalangeal (MCP) joint was spared clinically and radiologically (fig 1) despite the presence of an otherwise symmetrical mutilating arthropathy, in a 62 year old female patient with seropositive RA of 47 years duration. It was noted that the patient had worn one or more gold rings on her left ring finger throughout most of the time since the onset of her disease. It was suggested that gold molecules from her ring(s) might have passed through the skin and local lymphatic channels to allow sufficient gold to accumulate in the nearby MCP joint and thereby influence articular inflammation and erosion, as systemic chrysotherapy has been reported to do. ${ }^{5}$ This prospective study sought to investigate this hypothesis.

\section{Methods}

SUBJECTS

Patients with classic or definite RA, according to the American College of Rheumatology criteria $^{6}$ of greater than two years duration were recruited consecutively from a rheumatology outpatient clinic. Subjects were restricted to those who had never worn a gold ring (non-ring wearers) or who had worn a gold ring on the left ring finger throughout most of the time since the onset of their disease (ring wearers).

RADIOGRAPHIC ASSESSMENTS

Standard radiographs of the hands and wrists were obtained (with rings removed where possible) and were analysed using the Larsen method. ${ }^{7}$ The Larsen score at the five MCP, the four proximal interphalangeal (PIP), and the thumb interphalangeal joints in each hand was determined (GS). The mathematical difference between the total Larsen score in the right and the left hand of each patient was calculated and this represented the global difference between the two hands. A positive result reflected less articular damage, or sparing, of joints in the left hand.

\section{STATISTICAL METHODS}

Comparison was made between data from ring wearers and non-ring wearers, or between men and women, using the Mann-Whitney U test, and paired data from the right and left hand within each group were compared using the Wilcoxon signed rank test as appropriate. $p$ Values less than 0.05 were considered significant. 
Table 1 Larsen scores in individual hand joints in rheumatoid ring wearers and non-ring wearers. Values are median (25th-75th centile)

\begin{tabular}{|c|c|c|c|c|}
\hline & \multicolumn{2}{|c|}{ Ring wearers $(n=30)$} & \multicolumn{2}{|c|}{ Non-ring wearers $(n=25)$} \\
\hline & Left hand & Right hand & Left hand & Right hand \\
\hline \multicolumn{5}{|l|}{ Thumb } \\
\hline MCP & $(1-4)$ & $2(1-5)$ & $3(2-5)$ & $3(2-5)$ \\
\hline IP & $1 \quad(1-2)$ & $1(1-3)$ & $1(1-2)$ & $2(1-3.5)$ \\
\hline \multicolumn{5}{|c|}{ Index finger } \\
\hline MCP & $2.5(1-5)$ & $3(2-5)$ & $5(2-5)$ & $5(2-5)$ \\
\hline PIP & $2(1-3)$ & $2(1-3)$ & $2(1-4)$ & $2(1-4.25)$ \\
\hline \multicolumn{5}{|c|}{ Middle finger } \\
\hline MCP & $(1-2)$ & $2(1-5)$ & $4(2-5)$ & $4(1-5)$ \\
\hline PIP & $(1-3)$ & $2(1-4)$ & $2(2-5)$ & $2(1-4)$ \\
\hline \multicolumn{5}{|c|}{ Ring finger } \\
\hline MCP & $(1-2)$ & $1(1-5)$ & $4(1-5)$ & $2(1-5)$ \\
\hline PIP & $2 \quad(1-3)$ & $2(1-4)$ & $2(2-4.25)$ & $2(2-4.25)$ \\
\hline \multicolumn{5}{|c|}{ Little finger } \\
\hline MCP & $1.5(1-4)$ & $1(1-5)$ & $2(1-5)$ & $3(1-5)$ \\
\hline PIP & $2 \quad(1-3)$ & $2(1-4)$ & $2(1-5)$ & $3(1-5)$ \\
\hline
\end{tabular}

MCP, metacarpophalangeal joint; IP, interphalangeal joint; PIP, proximal interphalangeal joint.

\section{Results}

Fifty five subjects (39 female) were included in the study and all bar one had at least one erosion. Thirty patients (27 female) were ring wearers and 25 (12 female) were non-ring wearers. There was no significant difference in overall articular erosion in ring wearers compared with non-ring wearers: median (25th-75th centile) total Larsen scores was 44 $(24-71)$ in ring wearers and $53(36-79)$ in non-ring wearers $(p>0.05)$. However, analysis of data from individual joints in these patients revealed less articular erosion of the ring MCP joints of the left hand of ring wearers. In ring wearers, the Larsen score in the left hand ring MCP joint was significantly less than the right hand ring MCP joint of these patients ( $\mathrm{p}=$ 0.01) (table 1). A similar but less significant difference was also observed at an adjacent joint, between the left and right little MCP joint $(\mathrm{p}<0.05)$. However, no further significant difference was demonstrated at any other joint in the hand among ring wearers, nor at any joint in non-ring wearers $(\mathrm{p}>0.05)$ (table 1$)$. The Larsen score of the left hand ring MCP joint of ring wearers tended to be less than in the same joint of non-ring wearers $(\mathrm{p}=0.06)$ (table 1), with a similar but significant

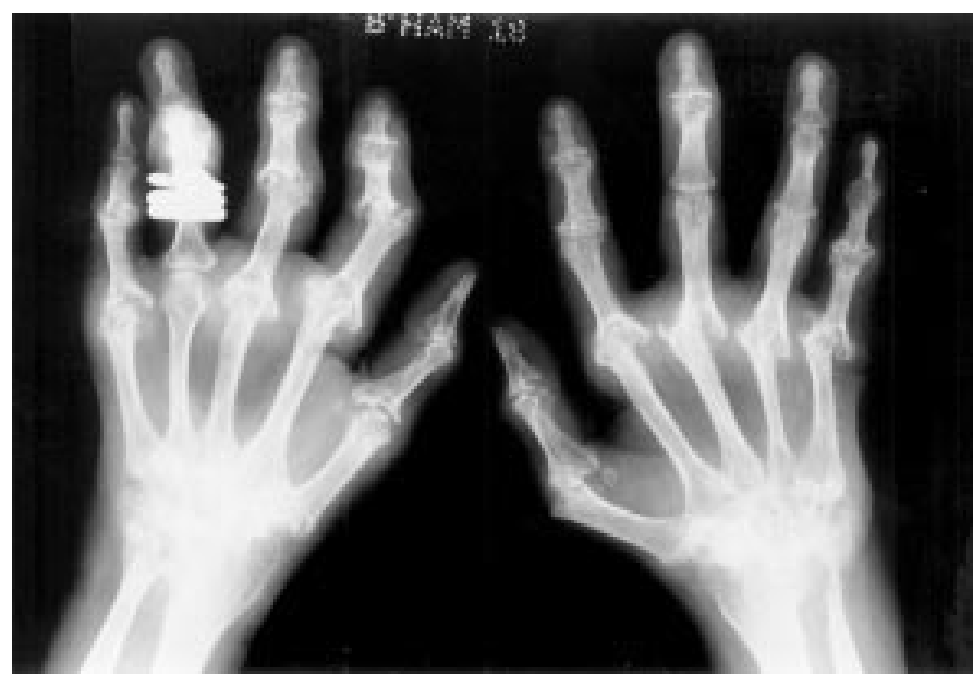

Figure 1 Radiological sparing of the left ring finger metacarpophalangeal joint despite an otherwise mutilating arthropathy in a 62 year old patient with longstanding $R A$ who had worn gold rings on her left ring finger throughout most of the time since disease onset. difference observed at the adjacent middle MCP joint of the left hand $(p=0.01)$. There were no significant differences between ring wearers and non-ring wearers at any other joints in the hand.

Median (25th-75th centile) global difference between left and right hand Larsen scores was $1(-1$ to +5$)$. Global difference data for ring wearers were not significantly different when compared with non-ring wearers, and were not significantly different between men and women.

\section{Discussion}

This study examined the hypothesis that gold molecules from a wedding ring might pass through skin and local lymphatic channels to the nearby middle, ring and little MCP joints in sufficient quantities to influence articular inflammation and erosion. The results suggested that there was less articular erosion, or sparing, of these left hand MCP joints of ring wearers when compared with their own equivalent right hand MCP joints or to the equivalent left hand MCP joints of non-ring wearers.

Gold has been used for the treatment of rheumatic disorders since the turn of the century and has been used with demonstrable effect in the treatment of RA since $1929 .{ }^{8}$ It is widely distributed throughout the body after chronic administration but in considerably varying concentrations. Concentrations of gold in tissues such as bone marrow, liver, and spleen are considerably higher than values in synovial membrane and cartilage, suggesting that comparatively small amounts of gold may be required at these sites to achieve a therapeutic effect. Gold has a wide variety of effects on elements of cellular and humoral immunity. However, the use of gold in the treatment of RA remains empirical, as its precise mechanism of action in these patients remains poorly understood. There is evidence that gold treatment can slow the rate of radiological progression in RA. ${ }^{5}$ Against this background, it is certainly possible that gold could accumulate in adjacent MCP joints in small but sufficient amounts to influence synovial inflammation at this site.

This study may not be the definitive epidemiological study on this subject and clearly has limitations. The predominance of females among ring wearers and of males among non-ring wearers is not surprising. An analysis confined to women only showed similar trends but sample sizes became too small to reach significance, and as sex did not seem to influence our findings, men were included in the complete analysis. The study did not examine the influence of handedness on articular erosion. One could speculate that different patterns of articular destruction between the right and left hand might simply reflect different patterns of use. However, it is of interest that previous studies have not shown a relation between hand dominance and articular deformity. ${ }^{9}$ In addition, one might expect handedness to produce global effects at most or all joints of the left hand. Clearly, the 
observed differences were confined to joints 'downstream' of the gold ring and there was little evidence of global sparing of the left hand, with only a very small median global difference favouring the left. It is also possible that the observed differences could simply reflect a local mechanical effect of the ring, unrelated to its gold content. However, despite these confounding factors, the preliminary findings presented provide support for the hypothesis that gold rings might influence local articular erosion and further studies are merited, which control for these factors and perhaps include a cohort wearing rings of a material other than gold.

We are grateful to Dr B McConkey, Dudley Road Hospital, Birmingham, for allowing us to describe his patient.

1 Harris ED. Rheumatoid arthritis: pathophysiology and implications for therapy. N Engl J Med 1990;322:1277-89.
2 Mulherin D, FitzGerald O, Bresnihan B. Clinical improvement and radiological deterioration in rheumatoid arthritis: evidence that the pathogenesis of synovial inflammation and articular erosion may differ. Br J Rheumatol 1996;35:1263-8.

3 Veale D, Farrell M, FitzGerald O. Mechanisms of joint sparing in a patient with unilateral psoriatic arthritis and a longstanding hemiplegia. Br J Rheumatol 1993;32:413-6.

4 Mulherin D, Bresnihan B, FitzGerald O. Digital denervation associated with absence of nail and distal interphalangeal joint involvement in psoriatic arthritis. J Rheumatol 1995;22:1211-2.

5 Iannuzzi L, Dawson N, Zein N, Kushner I. Does drug therapy slow radiographic deterioration in rheumatoid arthritis. N Engl J Med 1983;309:1023-8.

6 Ropes MW, Bennett GA, Cobb S, Jacox R, Jessar RA. 1958 revision of diagnostic criteria for rheumatoid arthritis. Bull Rheum Dis 1958;9:175-6.

7 Larsen A, Dale K, Eek M. Radiographic evaluation of rheumatoid arthritis and related conditions by standard reference films. Acta Radiol Diagn 1977;18:481-91.

8 Gordan DA. Gold compounds. In: Kelley WN, Harris ED, Ruddy S, Sledge CB, eds. Textbook of rheumatology, 3rd ed. Philadelphia: WB Saunders, 1989: 804-23.

9 Eberhardt K, Malcus Johnson P, Rydgren L. The occurrence and significance of hand deformities in early rheumatoid arthritis. Br J Rheumatol 1991;30:211-3. 